INRA Prod. Anim.,

2013, $26(5), 425-434$

\title{
Qualité des carcasses et de la viande des palmipèdes gavés
}

\author{
E. BAÉZA ${ }^{1}$, X. FERNANDEZ2,3,4, C. MARIE-ETANCELIN \\ 1 INRA, UR83 Recherches Avicoles, F-37380 Nouzilly, France \\ 2 INRA, UMR1289 TANDEM, F-31326 Castanet-Tolosan, France \\ ${ }^{3}$ Université de Toulouse, INPT ENSAT, UMR1289 TANDEM, F-31326 Castanet-Tolosan, France \\ ${ }^{4}$ Université de Toulouse, INPT ENVT, UMR1289 TANDEM, F-31076 Toulouse, France \\ 5 INRA, UR631 SAGA, F-31326 Castanet-Tolosan, France \\ Courriel : Elisabeth.Baeza@tours.inra.fr
}

En France, la production de viande de canard représente 13,4\% de la production totale de viande de volailles. Cette viande provient de deux filières distinctes, la filière dite " maigre » ou « du canard à rôtir » et la filière dite " grasse » ou du « canard gavé ». La viande issue de palmipèdes gavés présente des caractéristiques bien différentes de celle issue de palmipèdes non gavés, mais le consommateur n'est pas toujours informé de ces particularités.

La France est caractérisée par une consommation individuelle de viande de canard importante $(3,1 \mathrm{~kg} / \mathrm{hab} . / \mathrm{an})$ et supérieure à celle de la Chine $(1,9$ $\mathrm{kg} / \mathrm{hab}$./an) alors que ce pays est de loin le premier producteur mondial de viande de canard $(69 \%$ de la production mondiale), la France occupant la deuxième position avec une proportion bien moindre $(6 \%$ de la production mondiale). En France, la production de viande issue de canards gras était de $142720 \mathrm{t}$ en 2011 et représentait $59 \%$ du total des abattages contrôlés de canards (maigres et gras, CIFOG 2012). En 2010, la production de viande issue d'oies grasses était d'environ $720 \mathrm{t}$ sur un total de $2540 \mathrm{t}$ de viande d'oie (CIFOG 2012). La France se caractérise également par une production diversifiée de par les types de produits (canard à rôtir ou gavé), les systèmes de production (standard, Label rouge ou IGP Sud-Ouest) et les espèces ou génotypes utilisés (oies, canards de Barbarie, Pékin et mulard). Le canard mulard, hybride résultant du croisement entre un mâle Barbarie (Cairina moschata) et une femelle commune (Anas plathyrynchos) le plus souvent de type Pékin, représente cependant $97 \%$ des canetons mis en place (CIFOG 2012). Les carcasses des palmipèdes gras sont en général découpées : les magrets (noms donnés aux filets de canards gavés, muscles Pectoralis major) sont commercialisés soit sous forme de viande fraîche soit après transformation en magrets salés, séchés et/ou fumés, et les aiguillettes (muscles Pectoralis minor) sont plutôt valorisées en découpe fraîche. Les cuisses-pilons, les man- chons et les gésiers sont majoritairement commercialisés sous forme de confits. Le reste de la carcasse est utilisé pour la préparation de rillettes et la graisse est valorisée avec les confits et les rillettes (tableau 1). A l'issue du gavage qui dure en moyenne 12 jours (Litt 2010a), l'engraissement des animaux est fortement accru en particulier au niveau du foie et des tissus adipeux périphériques. La teneur en lipides des muscles est pratiquement doublée ce qui s'accompagne de fortes modifications du métabolisme et des caractéristiques physico-chimiques et structurales qui vont impacter la qualité nutritionnelle, sensorielle et technologique de la viande. Après avoir détaillé l'effet du gavage sur ces paramètres, nous décrirons différents facteurs de variation de la qualité des carcasses et de la viande des palmipèdes gavés.

Tableau 1. Produits transformés (en tonnes) issus des palmipèdes gras en France. Source : CIFOG 2012.

\begin{tabular}{|l|c|c|c|}
\hline & $\mathbf{2 0 0 3}$ & $\mathbf{2 0 0 7}$ & $\mathbf{2 0 1 1}$ \\
\hline Confits d'oie & 118 & 62 & 274 \\
\hline Confits de canard & 12733 & 16076 & 17901 \\
\hline Gésiers d'oie ou de canard confit & 984 & 1119 & 1121 \\
\hline Rillettes d'oie ou de canard & 496 & 454 & 504 \\
\hline Graisse d'oie ou de canard & 1176 & 1456 & 1483 \\
\hline Autres & 1493 & 1232 & 1556 \\
\hline
\end{tabular}

1 / Effet du gavage sur la composition corporelle, les caractéristiques physicochimiques, structurales et métaboliques des muscles et la qualité de la viande

Les canards gavés présentent un poids vif plus élevé que leurs contemporains non gavés du fait d'un fort accroissement du poids du foie (d'un facteur 8 à 9 chez le canard mulard) et de leur engraissement périphérique (gras souscutané et gras abdominal doublés, tableaux 2 et 3 ). Le poids des muscles de la cuisse n'est en général pas modifié par le gavage alors que le poids du muscle pectoral (filet ou magret sans peau) 
Tableau 2. Comparaison de la composition corporelle de canards de Barbarie mâles, âgés de 14 semaines, maigres ou gavés pendant 12 jours (Auvergne 1992).

\begin{tabular}{|l|c|c|}
\hline & Maigre & Gavé \\
\hline Poids vif (g) & $4045^{\mathrm{b}}$ & $4978^{\mathrm{a}}$ \\
\hline Rendement « prêt à cuire » (\% poids vif) & $62,8^{\mathrm{a}}$ & $55,9^{\mathrm{b}}$ \\
\hline Foie (\% poids vif) & $1,9^{\mathrm{b}}$ & $7,4^{\mathrm{a}}$ \\
\hline Gras abdominal (\% poids vif) & $1,4^{\mathrm{b}}$ & $3,8^{\mathrm{a}}$ \\
\hline Cuisses-pilons (\% poids vif) & 15,4 & 15,5 \\
\hline $\begin{array}{l}\text { Peau + gras sous-cutané des cuisses-pilons } \\
\text { (\% cuisses-pilons) }\end{array}$ & $21,7^{\mathrm{b}}$ & $39,7^{\mathrm{a}}$ \\
\hline Filets sans peau (\% poids vif) & $16,0^{\mathrm{a}}$ & $11,5^{\mathrm{b}}$ \\
\hline Peau des filets (\% poids vif) & $1,4^{\mathrm{b}}$ & $2,0^{\mathrm{a}}$ \\
\hline
\end{tabular}

$\mathrm{a}, \mathrm{b}$ : effet significatif du gavage à $\mathrm{P}<0,01$.

Tableau 3. Comparaison de la composition corporelle de canards de Barbarie et mulards mâles, âgés de 14 semaines, maigres ou gavés pendant 14 jours (moyenne $\pm E T, n=8$, Chartrin et al 2006a).

\begin{tabular}{|l|c|c|c|c|}
\hline & \multicolumn{2}{|c|}{ Barbarie } & \multicolumn{2}{c|}{ Mulard } \\
\hline & Maigre & Gavé & Maigre & Gavé \\
\hline Poids vif (g) & $5418 \pm 245^{\mathrm{b}}$ & $6393 \pm 441^{\mathrm{a}}$ & $4455 \pm 392^{\mathrm{b}}$ & $6473 \pm 351^{\mathrm{a}}$ \\
\hline Foie (g) & $77 \pm 7^{\mathrm{b}}$ & $467 \pm 90^{\mathrm{a}}$ & $61 \pm 13^{\mathrm{b}}$ & $494 \pm 97^{\mathrm{a}}$ \\
\hline Gras abdominal (g) & $109 \pm 20^{\mathrm{b}}$ & $220 \pm 23^{\mathrm{a}}$ & $73 \pm 35^{\mathrm{b}}$ & $224 \pm 42^{\mathrm{a}}$ \\
\hline Filet sans peau $(\mathrm{g})$ & $398 \pm 39$ & $408 \pm 49$ & $278 \pm 30$ & $309 \pm 28$ \\
\hline
\end{tabular}

a, b : pour un génotype donné, effet significatif du gavage à $P<0,001$. peut être, soit inchangé, soit affecté (tableaux 2 et 3 ). En effet, au cours de la première semaine de gavage, l'apport unique de maïs se traduit par un déficit de protéines alimentaires et les protéines du muscle pectoral peuvent alors être mobilisées et utilisées pour contribuer à l'hyperplasie et l'hypertrophie du foie. Ainsi, Chartrin et al (2007) avait enregistré pour des canards mulards des poids de muscle pectoral de $307 \mathrm{~g}$ à la mise en gavage, $272 \mathrm{~g}$ après une semaine de gavage et $304 \mathrm{~g}$ à l'issue de deux semaines de gavage. Pour des canards mulards contemporains, nourris à volonté, les poids de muscle pectoral, aux mêmes âges, étaient de 309, 311 et 314 g, respectivement.

La teneur en lipides des muscles est également doublée en réponse au gavage (tableau 4) au détriment de la teneur en eau (Chartrin et al 2006a). Ce dépôt de lipides est essentiellement dû à une accumulation de triglycérides dont l'afflux au muscle est consécutif à une lipogenèse de novo au niveau hépatique suite au gavage puis à leur exportation via les VLDL («Very Low Density Lipoproteins », Baéza et al 2005). La composition en acides gras est aussi modifiée avec un enrichissement en Acides Gras MonoInsaturés (AGMI) en particulier en acide oléique (C18:1) au détriment des Acides Gras Poly-Insaturés (AGPI). Le magret de canard présente donc une qualité nutritionnelle bien différenciée de celle du filet de canard nourri à volonté. Ces lipides vont s'accumuler de façon préférentielle dans des adipocytes, dont la surface relative sur des coupes transversales de muscle est pratiquement doublée, mais également dans les fibres musculaires (Zanusso et al 2002, Chartrin

Tableau 4. Comparaison de la teneur et de la composition en lipides totaux ( $\mathrm{g} / 100 \mathrm{~g}$ muscle) et en acides gras (\% AG totaux) de filets et magrets de canards de Barbarie et mulards mâles, âgés de 14 semaines, maigres ou gavés pendant 14 jours (moyenne $\pm E T, n=8$, Chartrin et al 2006a).

\begin{tabular}{|l|c|c|c|c|}
\hline \multirow{2}{*}{} & \multicolumn{2}{|c|}{ Barbarie } & \multicolumn{2}{c|}{ Mulard } \\
\cline { 2 - 5 } & Maigre & Gavé & Maigre & Gavé \\
\hline Lipides totaux & $2,26 \pm 0,36^{\mathrm{b}}$ & $3,65 \pm 0,51^{\mathrm{a}}$ & $3,13 \pm 0,54^{\mathrm{b}}$ & $5,24 \pm 0,96^{\mathrm{a}}$ \\
\hline Triglycérides & $1,02 \pm 0,24^{\mathrm{b}}$ & $2,37 \pm 0,47^{\mathrm{a}}$ & $1,79 \pm 0,32^{\mathrm{b}}$ & $3,66 \pm 0,11^{\mathrm{a}}$ \\
\hline Cholestérol & $0,12 \pm 0,04$ & $0,13 \pm 0,05$ & $0,13 \pm 0,03$ & $0,16 \pm 0,04$ \\
\hline Phospholipides & $1,13 \pm 0,10$ & $1,15 \pm 0,17$ & $1,21 \pm 0,24$ & $1,42 \pm 0,11$ \\
\hline AGS $^{*}$ & $39,20 \pm 1,59$ & $37,97 \pm 1,22$ & $36,92 \pm 1,82$ & $37,91 \pm 1,19$ \\
\hline AGMI $^{*}$ & $37,20 \pm 2,24^{\mathrm{b}}$ & $46,35 \pm 1,61^{\mathrm{a}}$ & $42,22 \pm 3,18^{\mathrm{b}}$ & $45,66 \pm 0,92^{\mathrm{a}}$ \\
\hline AGPI $^{*}$ & $23,60 \pm 1,86^{\mathrm{a}}$ & $15,69 \pm 1,39^{\mathrm{b}}$ & $20,86 \pm 3,27^{\mathrm{a}}$ & $16,43 \pm 1,32^{\mathrm{b}}$ \\
\hline
\end{tabular}

$a, b$ : pour un génotype donné, effet significatif du gavage à $P<0,01$.

*AGS, AGMI, AGPI = Acides Gras Saturés, Mono-Insaturés et Poly-Insaturés. 
et al 2005). Cet afflux de lipides va modifier l'orientation du métabolisme énergétique musculaire. Dans le muscle pectoral des canards de Barbarie et mulards, l'activité de la lactate déshydrogénase, marqueur du métabolisme glycolytique, est peu modifiée. En revanche, celle de la $\beta$-hydroxyacyl déshydrogénase, une des enzymes de l'oxydation des acides gras, est accrue (Zanusso et al 2002, Chartrin et al 2006b). L'enrichissement en lipides des muscles s'accompagne donc d'une capacité supérieure d'oxydation des acides gras. Toutefois, ni la taille, ni la typologie des fibres musculaires ne sont modifiées par le gavage (Zanusso et al 2002, Chartrin et al 2005). En revanche, Zanusso et al (2002) ont mis en évidence une diminution de la teneur en collagène $(18,9 v \mathrm{~s}$ $23,9 \%$ des protéines totales) et de sa solubilité $(11,2$ vs 18,9\%) dans les magrets de canards de Barbarie gavés comparés à des filets de canards de Barbarie du même âge, nourris à volonté.

L'ensemble de ces modifications induites par le gavage vont avoir des répercussions sur la qualité technologique et sensorielle de la viande. Le magret présente une couleur plus claire et plus brun chocolat que le filet. Ceci se traduit par des valeurs de luminance ( $\mathrm{L}^{*}$ ) et d'indice de jaune $\left(b^{*}\right)$ plus élevées (tableau 5, Chartrin et al 2006c). En effet, le maïs est riche en pigments xanthophylles qui sont liposolubles et qui vont accentuer la coloration jaune de la viande. L'indice de rouge $\left(\mathrm{a}^{*}\right)$ lié à la teneur en pigments héminiques de la viande n'est pas affecté par le gavage. Le magret a des pertes à la cuisson et une note de flaveur plus élevées, mais une note de jutosité plus faible que le filet. Le gavage n'a pas d'effet sur la tendreté de la viande (Chartrin et al 2006c). Le stockage accru de substrats énergétiques dans le muscle semble concerner non seulement les lipides mais également le glycogène, le $\mathrm{pH}$ ultime des magrets étant inférieur à celui des filets $(5,81$ vs 5,88 données non publiées) ce qui pourrait expliquer, en partie, leur luminance avant cuisson et leurs pertes en jus après cuisson plus élevées. La teneur en lipides élevée du magret associée à une teneur importante en pigments héminiques, riches en fer, devrait favoriser la sensibilité à l'oxydation de cette viande lors de sa conservation à $+4^{\circ} \mathrm{C}$ et $-20^{\circ} \mathrm{C}$ et/ou de sa transformation. Au-delà de l'impact direct du gavage sur la qualité de la viande de canard, l'itinéraire technique des phases d'élevage et de gavage va aussi influencer ces paramètres.

\section{2 / Effet de la conduite en élevage}

En début de croissance, les animaux sont nourris à volonté. Puis intervient une phase de rationnement qui a pour objectif d'entraîner les canards et les oies qui seront gavés par une alimentation sous forme de repas avec une prise alimentaire sur un temps très court. Ce rationnement peut être soit quantitatif, soit horaire et son niveau doit être adapté à la saison, les besoins d'entretien des animaux variant selon la température extérieure lors de leur séjour quotidien sur les parcours (AGPM 2000).

\section{1 / Caractéristiques de l'aliment en phase d'élevage}

Quelle que soit la saison, prolonger la durée d'utilisation en phase d'élevage d'un aliment " démarrage » riche en protéines $(18,6 \%)$ de 2 à 4 ou 6 semaines n'a pas d'effet sur l'engraissement de la carcasse et sur le poids des muscles mesurés en fin de gavage (Robin et Castaing 1993). Cependant, une substitution du maïs par du blé en phase d'élevage induit une diminution du poids du magret avec aiguillette (340 g vs $312 \mathrm{~g}$ lorsque la substitution est totale, $\mathrm{P}<0,05$ ), mais n'a pas d'effet sur l'engraissement corporel (Castaing et Robin 1996). La substitution du maïs par du sorgho en phase d'élevage n'a pas d'effet sur la composition corporelle des oies à l'issue du gavage (Arroyo 2012).

La concentration énergétique de l'aliment (2 850 vs $3150 \mathrm{kcal} / \mathrm{kg}$ d'aliment) en phase d'élevage n'a pas d'effet sur la composition corporelle des oies après gavage (Leprettre et al 1998). Pour le canard mulard, les résultats sont différents : Robin et Castaing (1998a, 1998b) et Castaing et al (2000) ont montré que l'utilisation en phase d'élevage d'un aliment à $3000 \mathrm{kcal} / \mathrm{kg}$ permettait d'obtenir, à l'issue du gavage, des poids de muscles et de gras sous-cutané supérieurs par comparaison avec l'utilisation d'un aliment à $2750 \mathrm{kcal} / \mathrm{kg}$. Le rendement technologique des magrets séchés était également plus élevé. De l'analyse sensorielle des magrets séchés il ressortait que la viande était plus suintante et moins dure à mastiquer et que l'odeur globale et les goûts de viande de canard et de viande crue étaient moins prononcés.

\section{2 / Conduite du rationnement}

Dubois et al (1996) ont montré qu'en période hivernale, le poids vif et le poids de muscle pectoral étaient plus importants $(7,5$ vs $7,3 \mathrm{~kg}$ et 274 vs $258 \mathrm{~g}$, $\mathrm{P}<0,05)$ lorsque les oies étaient soumises à un rationnement quantitatif correspondant à $90 \%$ des besoins théoriques par comparaison avec un rationnement horaire (accès à l'aliment pendant $2 \mathrm{~h}$ par jour).

\section{3 / Densité des animaux en phase d'élevage}

Elever les animaux avec des densités élevées permet d'améliorer les performances technico-économiques; cependant, cela peut avoir des répercussions sur la croissance des animaux. Leprettre et al (2000a) ont ainsi montré que des oies élevées à une densité de $3,5 / \mathrm{m}^{2}$ avaient un poids vif et un poids de magret supérieurs $(+362 \mathrm{~g}$ et $+30 \mathrm{~g}$, respectivement) à ceux d'oies élevées à une densité de $5 / \mathrm{m}^{2}$ du fait d'une compétition

Tableau 5. Qualité technologique de filets et magrets de canards de Barbarie et mulards mâles, âgés de 14 semaines, maigres ou gavés pendant 14 jours (moyenne $\pm E T, n=20$, Chartrin et al 2006c).

\begin{tabular}{|l|c|c|c|c|}
\hline \multirow{2}{*}{} & \multicolumn{2}{|c|}{ Barbarie } & \multicolumn{2}{c|}{ Mulard } \\
\cline { 2 - 5 } & Maigre & Gavé & Maigre & Gavé \\
\hline Luminance $\left(\mathrm{L}^{*}\right)$ & $35,0 \pm 2,1^{\mathrm{b}}$ & $43,6 \pm 3,1^{\mathrm{a}}$ & $31,9 \pm 1,8^{\mathrm{b}}$ & $41,2 \pm 3,7^{\mathrm{a}}$ \\
\hline Indice de rouge $\left(\mathrm{a}^{*}\right)$ & $13,6 \pm 0,9$ & $12,8 \pm 0,9$ & $13,8 \pm 1,0$ & $14,2 \pm 1,2$ \\
\hline Indice de jaune $\left(\mathrm{b}^{*}\right)$ & $11,4 \pm 1,1^{\mathrm{b}}$ & $14,7 \pm 2,0^{\mathrm{a}}$ & $8,9 \pm 1,4^{\mathrm{b}}$ & $14,3 \pm 2,0^{\mathrm{a}}$ \\
\hline Pertes à la cuisson $(\%$ poids frais$)$ & $14,6 \pm 1,5^{\mathrm{b}}$ & $17,0 \pm 1,9^{\mathrm{a}}$ & $14,5 \pm 1,6^{\mathrm{b}}$ & $15,9 \pm 1,5^{\mathrm{a}}$ \\
\hline
\end{tabular}

$\mathrm{a}, \mathrm{b}$ : pour un génotype donné, effet significatif du gavage à $\mathrm{P}<0,001$. 
moins importante entre animaux pour l'accès à l'aliment.

\section{3 / Effet de la conduite en gavage}

\section{1 / Présentation et composition de l'aliment de gavage}

En gavage, le maïs peut être présenté sous la forme de grains entiers et/ou broyés. Chez l'oie, l'association des deux formes de présentation permet d'obtenir un poids vif plus élevé qu'avec chacune des formes de présentation (Dubois et al 1996). Le poids de muscle pectoral était moins élevé pour les oies gavées avec $100 \%$ de maïs broyé. Le mélange optimal serait composé de $42 \%$ de maïs en grains entiers et $58 \%$ de farine de maîs (Dubois et al 2000). En revanche, chez le canard mulard, Guy et al (2000) n'ont pas mis en évidence d'effet significatif de la forme de présentation du maïs sur le poids de muscle pectoral ni sur l'engraissement abdominal et souscutané.

Il existe plusieurs variétés de maïs et différents modes de conservation. Les grains dentés ont une teneur en amidon supérieure à celle des grains cornés dentés qui sont eux plus riches en matières azotées. Chez le canard mulard, l'utilisation de maïs corné ou corné denté en gavage ne modifie pas le poids du muscle pectoral ni la proportion de gras souscutané (Robin et Castaing 2000). L'utilisation de maïs "waxy », dont l'amidon est presque exclusivement composé d'amylopectine, n'a pas non plus eu d'effet sur le poids du muscle pectoral ni sur la proportion de gras sous-cutané par comparaison avec l'utilisation d'un maïs classique qui contient $70-75 \%$ d'amylopectine et 25-30\% d'amylose (Castaing et Robin 2000). Le maïs utilisé pour le gavage peut être conservé soit sous forme sèche (teneur en eau de $15 \%$ ), soit sous forme humide par inertage en silo souple (teneur en eau de $31 \%$ ). La composition corporelle des canards mulards gavés n'est pas affectée par le mode de conservation du maïs (Ducamp et al 2008, Peillod et al 2010). La variété de maïs utilisée en gavage et sa forme de conservation n'ont donc pas d'effet sur la composition corporelle des canards.

La substitution complète du maïs par du sorgho en phases d'élevage puis de gavage n'a pas d'effet sur le poids, les paramètres de la couleur $\left(\mathrm{L}^{*}, \mathrm{a}^{*}, \mathrm{~b}^{*}\right)$, la composition chimique, le profil en acides gras et le rendement après salageséchage du magret d'oie, alors que le poids du foie est accru (Arroyo 2012). L'évaluation hédonique des magrets salés séchés n'a pas mis en évidence de différence entre les deux types de céréales.

De l'huile peut être incorporée au maïs lors du gavage pour servir de lubrifiant. De fait, la composition en acides gras et donc la qualité nutritionnelle et l'aptitude à la conservation de la graisse et de la viande peuvent être modifiées selon la nature de l'huile utilisée. Gabarrou et al (2000) ont gavé des canards mulards en ajoutant au maïs de l'huile de noix ou de colza : les teneurs respectives en eau, lipides et protéines du muscle pectoral et du tissu adipeux sous-cutané n'ont pas été affectées par la source de lipides alimentaires. En revanche, les huiles de colza et de noix augmentent la teneur en acide linolénique (C18:3) dans le muscle pectoral et la graisse sous-cutanée par rapport à un régime de maïs seul. $\mathrm{La}$ teneur en acide linoléïque (C18:2) est uniquement accrue avec l'huile de noix. Peyhorgue et al (2002b) ont comparé un apport d'huile de maïs ou de noix dans la ration de gavage à hauteur de $4 \%$ par rapport à un régime de maïs seul. L'apport

Figure 1. Effet de la durée du gavage (2 repas quotidiens) sur le poids de foie, de muscles et de gras sous-cutané et abdominal de canards mulards (Sazy et al 1993).
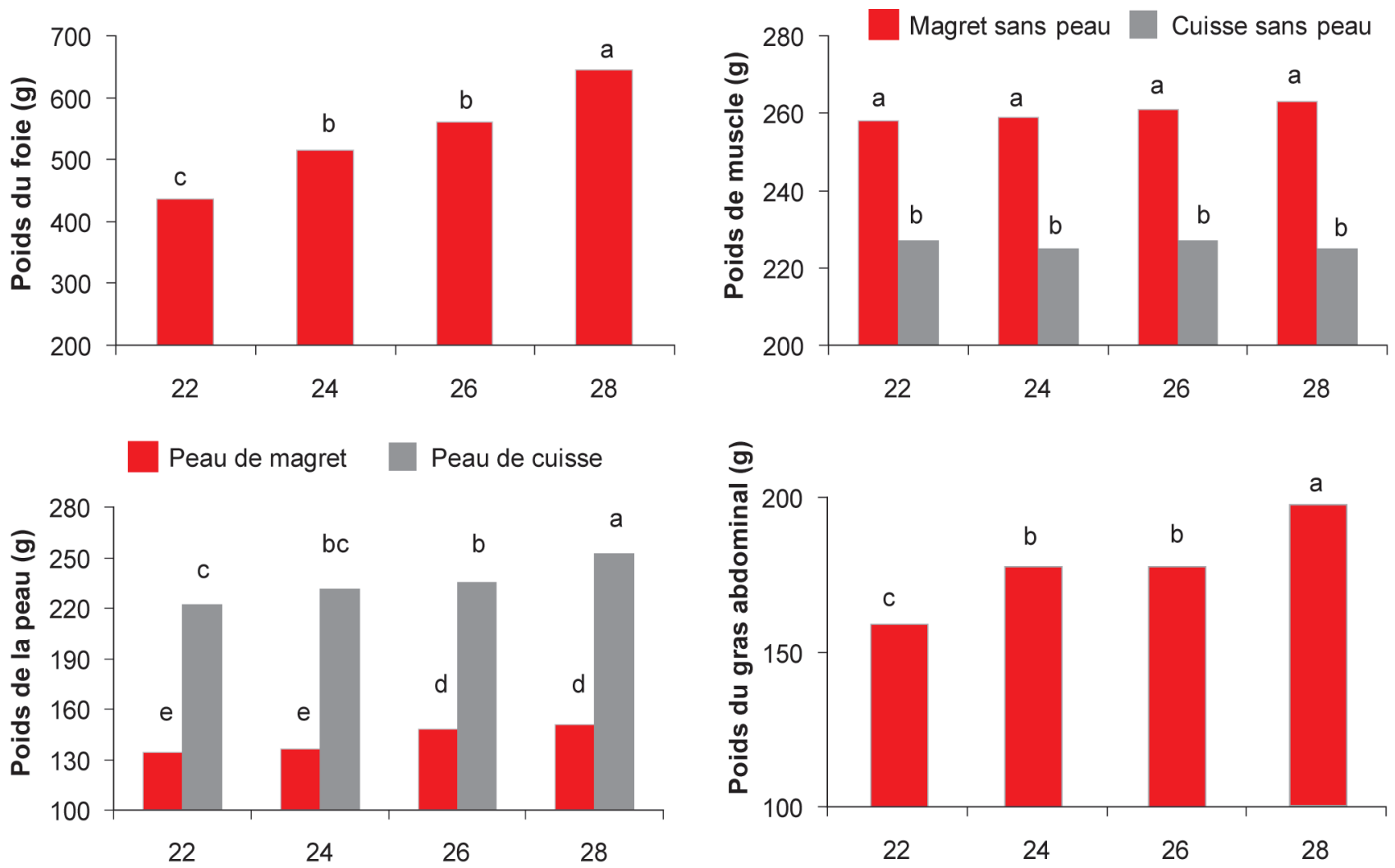

Nombre de repas de gavage

Nombre de repas de gavage

$a, b, c, d, e$ : effet significatif du nombre de repas de gavage à $\mathrm{P}<0,05$ 
d'huile augmente la teneur en C18:2 dans le magret de canard mulard $(+50 \%$ pour la peau et $+30 \%$ pour le muscle). Avec l'huile de noix, la teneur en C18:3 est multipliée par 3 pour les deux compartiments, peau et muscle. L'incorporation d'huile dans la ration de gavage ne modifie pas le rendement après séchage des magrets. En revanche, elle confère une couleur plus jaune et un aspect plus huileux au gras, un aspect plus suintant et une odeur de gras plus marquée à la tranche de magret séché. Elle n'a aucun effet sur le goût et la texture.

Enfin, chez le canard, une supplémentation du maïs broyé avec des protéines (44 g de poudre de lait/kg de maïs) ou avec de l'amidon ( $24 \mathrm{~g} / \mathrm{kg}$ maïs) n'a aucun effet sur le poids du magret et du tissu adipeux sous-cutané (Robin et Castaing 1998c).

\section{2 / Age à la mise en gavage}

Réduire la durée d'élevage avant la mise en gavage permet de diminuer les coûts de production, mais cela affecte le développement corporel, en particulier le développement des muscles pectoraux qui est tardif chez les oiseaux par comparaison à celui des muscles des cuisses et des pilons. Leprettre et al (2000b) ont montré que des oies mises en gavage à l'âge de 18 semaines avaient un poids vif à l'abattage, un poids de magret sans peau et un poids de peau de magret supérieurs à ceux des oies mises en gavage à l'âge de 12 semaines (respectivement, 9,52 vs 8,98 kg, 297 vs $269 \mathrm{~g}$ et 246 vs $216 \mathrm{~g}$ ), alors que le poids de foie n'était pas modifié (964 vs $961 \mathrm{~g}$ ). De plus, les magrets des oies mises en gavage à 18 semaines étaient plus riches en protéines et de teinte plus foncée que ceux des oies mises en gavage à 12 semaines. Le rendement des magrets après séchage $(65,5$ vs $62,7 \%)$ et les notes obtenues lors de l'analyse sensorielle pour l'aspect et la texture étaient aussi supérieurs pour les oies gavées tardivement (Auvergne et al 1998, Babilé et al 2000). De façon cohérente, les canards mulards mis en gavage à 13 semaines ont un poids vif et des poids de foie, de magret sans peau et de peau du magret plus élevés que des canards mis en gavage à 11 semaines (Castaing et al 2000). Leurs magrets sont aussi de teinte plus foncée, plus jaune et ils sont jugés plus tendres. Ainsi, réduire l'âge à la mise en gavage a globalement un effet négatif sur les performances des palmipèdes.

\section{3 / Durée du gavage}

Plus la durée de gavage est élevée, plus le poids de foie est accru et plus l'engraissement sous-cutané et abdominal est important alors que le poids des muscles n'est pas modifié chez le canard (figure 1, Sazy et al 1993, Robin et Castaing 1998b). Les teneurs en eau, protéines et lipides du magret ne sont pas modifiées lorsque la durée de gavage s'accroît de 9 à 15 jours, alors que le rendement en séchage augmente de 75,2 à 77,4\% (Peyhorgue et al 2002a). Avec une durée de gavage de 15 jours $v s 9$ jours, les magrets séchés sont jugés moins secs, plus tendres, plus fondants et présentent une odeur de gras plus développée. De même, après cuisson au grill, les magrets produits par des canards gavés pendant 15 jours sont jugés plus tendres, plus fondants et plus juteux que ceux issus de canards gavés pendant 9 jours seulement (Peyhorgue et al 2002a).

\section{4 / Logement en gavage}

Robin et al (2000) ont comparé les performances de gavage de canards mulards placés en cages individuelles, en épinettes collectives de 5 canards ou en parcs collectifs de 15 canards : le type de logement n'a eu aucun effet sur la composition corporelle et les caractéristiques physico-chimiques des magrets. Sur le plan de la présentation des carcasses, les animaux gavés en logements collectifs présentent davantage de griffures sur les cuisses que ceux gavés en cages individuelles (Litt 2010b).

\section{4 / Effet des conditions de pré-abattage et de la tech- nologie d'abattage}

La mise à jeun des animaux avant abattage est nécessaire pour préserver la qualité sanitaire des carcasses, mais un jeûne prolongé peut entraîner une diminution du poids du foie gras. Chez le canard de Barbarie gavé, lorsque la durée de jeûne est de 13 ou 19 h, la perte de poids vif est respectivement, de 134 ou $248 \mathrm{~g}$ et la perte de poids du foie est de 52 ou $75 \mathrm{~g}$ (Baudonnet-Lenfant et al 1991). Chez le canard mulard âgé de 12 semaines, la perte de poids est de 0,28 ou $0,35 \%$ par heure de jeûne, respectivement, selon qu'il y a accès ou non à un abreuvement (Sazy et Héraut 1996b). Il est donc conseillé d'éviter des durées de jeûne avant abattage supérieures au délai minimum imposé par la législation sanitaire soit $8 \mathrm{~h}$.

Fernandez et al (2011) ont étudié l'effet de la durée du transport (30 vs 150 min) et de l'attente sur quai avant abattage (15 vs $120 \mathrm{~min}$ ) sur la qualité du foie gras et du magret de canards mulards. Ils n'ont pas mis en évidence d'effet de ces facteurs sur le poids vif et le poids de foie. De même, le stock de glycogène intramusculaire et la cinétique post-mortem du $\mathrm{pH}$ de la viande n'étaient pas affectés. En revanche, la résistance de la viande crue à une force de compression augmentait avec la durée d'attente sur quai (47,8 vs 37,8 $\mathrm{N} / \mathrm{cm}^{2}$ ). Fernandez et al (2010) ont comparé différentes techniques de narcose lors de l'abattage d'oies et de canards mulards gavés : électronarcose en bain (50 Hz en courant alternatif, $130 \mathrm{~mA}, 4 \mathrm{~s}$ ), électronarcose bitemporale $(50 \mathrm{~Hz}$ en courant alternatif, $600 \mathrm{~mA}, 4 \mathrm{~s}$ ), étourdissement mécanique avec un pistolet à tige perforante et étourdissement en atmosphère modifiée (phase $1,40 \% \mathrm{CO}_{2}$ - $30 \% \mathrm{O}_{2}-30 \% \mathrm{~N}_{2}$ ) pendant 2 min suivie de la phase $2, \mathrm{CO}_{2}>85 \%$ dans l'air pendant $2 \mathrm{~min}$ ). Chez les deux espèces, l'étourdissement en atmosphère modifiée était associé à une vitesse de chute du $\mathrm{pH}$ post-mortem plus lente et à une moindre accumulation d'acide lactique dans le magret. Chez les canards mulards, la clarté des magrets était moindre pour les techniques qui tuent les animaux avant la saignée (électronarcose en bain et gaz). La texture, l'exsudat lors de la conservation au froid des magrets crus et les caractéristiques sensorielles des magrets cuits n'étaient pas affectés par la technique de narcose.

\section{5 / Effet de l'espèce, de la souche et du sexe}

Les souches d'oies (landaises, polonaises et italiennes) présentent des aptitudes au gavage très différentes. En tenant compte des différences de gabarit entre souches, l'oie landaise est la plus performante pour produire du foie gras et dépose moins de gras abdominal et sous-cutané que les oies polonaises et italiennes (Poujardieu et al 1994a). Une seule étude a été réalisée pour comparer les performances de croissance et de gavage de l'oie landaise et de canards de Barbarie et mulards élevés et gavés de façon contemporaine et dans les mêmes conditions (tableau 6, Guy et al 1995). Les oies landaises ont un poids vif et des poids de foie et de muscles au niveau de la cuisse supérieurs à ceux des canards. Les canards de Barbarie ont un poids de foie inférieur à celui des canards mulards (Guy et al 1995, Guy et al 1999), mais il est possible d'atteindre des poids de foie similaires pour les deux types de canards (Baéza et al 2005). En revanche, le canard de Barbarie présente l'engraissement sous-cutané et la teneur en lipides du magret les plus faibles et un rendement en viande plus important que le canard mulard (Guy et al 1999). Cette observation a été confirmée par Chartrin et al (2006a), avec un dépôt de triglycérides dans le muscle plus important chez le canard mulard (tableaux 3 et 4). La surface occupée par les adipocytes sur une coupe transversale de muscle 
Tableau 6. Comparaison de la composition corporelle d'oies landaises et de canards de Barbarie et mulards gavés (Guy et al 1995).

\begin{tabular}{|l|c|c|c|}
\hline \multirow{2}{*}{} & \multirow{2}{*}{ Oies } & \multicolumn{2}{|c|}{ Canards } \\
\cline { 3 - 4 } & & Mulard & Barbarie \\
\hline Poids vif (g) & $7444^{\mathrm{a}}$ & $6387^{\mathrm{b}}$ & $6314^{\mathrm{b}}$ \\
\hline Foie (g) & $793^{\mathrm{a}}$ & $702^{\mathrm{b}}$ & $560^{\mathrm{c}}$ \\
\hline Peau de la cuisse (g) & $251^{\mathrm{a}}$ & $254^{\mathrm{a}}$ & $172^{\mathrm{b}}$ \\
\hline Muscle de la cuisse (g) & $268^{\mathrm{a}}$ & $204^{\mathrm{c}}$ & $228^{\mathrm{b}}$ \\
\hline Peau du magret (g) & $136^{\mathrm{a}}$ & $133^{\mathrm{a}}$ & $101^{\mathrm{b}}$ \\
\hline Muscle du magret (g) & $214^{\mathrm{c}}$ & $273^{\mathrm{b}}$ & $318^{\mathrm{a}}$ \\
\hline Teneur en lipides du magret (\% MS) & $32,1^{\mathrm{a}}$ & $26,3^{\mathrm{b}}$ & $19,4^{\mathrm{c}}$ \\
\hline
\end{tabular}

$a, b, c$ : effet significatif de l'espèce à $P<0,001$.

est d'ailleurs supérieure chez le canard mulard $(3,52$ vs $1,98 \%$ dans le muscle Pectoralis major et 3,58 vs 2,40\% dans le muscle Sartorius de la cuisse, Chartrin et al 2005). Cependant, il n'a pas été mis en évidence de différence significative entre canards de Barbarie et mulards gavés pour ce qui concerne le métabolisme énergétique du magret et sa qualité sensorielle à l'exception de la tendreté jugée supérieure pour les canards mulards (Chartrin et al 2006c).

La souche de la cane commune, mère du canard mulard, affecte aussi la qualité de la viande du canard mulard gavé (Setiawan 1994). Ainsi, à 12 semaines d'âge, les magrets de canards mulards issus de canes Pékin sont significativement plus riches en lipides intramusculaires $(3,1 \%)$ que ceux issus de canes Tsaiya $(2,1 \%)$ - cane commune chinoise -, les canards mulards de mères croisées étant intermédiaires. Si aucune différence n'apparaît en termes de taux de matière azotée totale ou de minéraux dans le muscle, en revanche les magrets des canards mulards issus de canes Tsaiya sont moins rouges que ceux des autres souches.

Plusieurs souches commerciales d'oies landaises et de canards mulards sont dis- ponibles sur le marché. Selon l'itinéraire d'élevage et de gavage mis en œuvre, ces souches ne présenteront cependant pas les mêmes caractéristiques de composition corporelle et de qualité de viande (tableau 7, Sazy et Héraut 1996a, Castaing et al 2000, Robin et al 2002, Auvergne et al 2002).

Il existe un dimorphisme sexuel sur le poids vifs des canards, les mâles étant plus lourds que les femelles : de l'ordre de $55 \%$ chez le canard de Barbarie, de $65 \%$ chez le canard Hinny (Tai et Rouvier 1998). Chez l'oie, ce dimorphisme est seulement de 92\% (Leprettre et al 200a). Cependant l'effet du sexe sur la qualité de la viande produite n'a été étudié que chez l'oie, car il est interdit en France de gaver les canes en raison de défauts de qualité plus importants sur leurs foies (50-55\% de foies déclassés du fait d'un réseau de veines trop important, données non publiées). Les oies mâles ont en général un poids vif et des poids de foie, de muscles, de gras sous-cutané et abdominal plus importants que les femelles (Dubois et al 1993 et 1996, Leprettre et al 2000a). Après prise en compte des différences de gabarit entre animaux, les oies femelles ont toujours un poids de gras abdominal et un poids de muscle de la cuisse plus faibles, mais un engraissement sous-cutané au niveau du magret et de la cuisse plus marqué que les mâles (Poujardieu et al 1994a).

\section{6 / Déterminisme génétique de la qualité des carcasses et de la viande de palmipèdes gavés}

Chez l'oie gavée, aucune étude génétique n'a porté sur la qualité de la viande et seule la qualité de la carcasse a été explorée. Ainsi, Larzul et al (2000) et Richard et al (2006) ont estimé une héritabilité (pourcentage de la variation du caractère attribué à la génétique) élevée du poids du paletot (ensemble composé de la peau, de la graisse et de la viande) d'oies polonaises et landaises gavées (respectivement 0,58 et 0,85 ). Le poids du paletot est très corrélé au poids vifs des animaux à 8 semaines d'âge ( $+0,88$ et $+0,82$ respectivement), mais indépendant du poids de foie gras.

Chez le canard mulard, la sélection d'un caractère spécifique du mulard gavé doit être réalisée par sélection des lignées parentes (soit Barbarie, soit commune, soit les deux) (Marie-Etancelin et al 2008). Il est donc indispensable de bien connaître le déterminisme génétique des caractères du mulard qui pourront être sélectionnés, ensuite, dans les populations parentes. Le poids du magret de canard présente une valeur d'héritabilité moyenne : 0,27 pour le canard de Barbarie (Babilé 1989) et variant de 0,17 à 0,22 dans la voie maternelle du canard mulard (Poujardieu et al 1994b, Chapuis et Larzul 2008). Les estimations d'héritabilité de performances du canard mulard sont quasi systématiquement plus faibles dans la voie paternelle (Barbarie) que dans la voie maternelle (commune) (Chapuis et Larzul 2008, Marie-Etancelin et al 2010). Lorsque le muscle du magret est séparé de la « peau + gras » du magret, le poids du compartiment musculaire est plus héritable (environ 0,30 dans la voie commune)

Tableau 7. Composition corporelle de canards mulards mâles gavés issus de 4 croisements différents (Robin et al 2002).

\begin{tabular}{|l|c|c|c|c|}
\hline & SL2 $\times$ Dominant & PKLc $\times$ MMg & M12ng $\times$ SR60 & M16 $\times$ SR60 \\
\hline Foie (g) & $654^{\mathrm{c}}$ & $718^{\mathrm{b}}$ & $708^{\mathrm{b}}$ & $741^{\mathrm{a}}$ \\
\hline Peau magret et cuisse-pilon (g) & $506^{\mathrm{c}}$ & $575^{\mathrm{a}}$ & $534^{\mathrm{b}}$ & $526^{\mathrm{bc}}$ \\
\hline Gras abdominal (g) & $239^{\mathrm{b}}$ & $270^{\mathrm{a}}$ & $262^{\mathrm{a}}$ & $245^{\mathrm{b}}$ \\
\hline Magret sans peau (g) & $328^{\mathrm{a}}$ & $318^{\mathrm{a}}$ & $292^{\mathrm{b}}$ & $277^{\mathrm{c}}$ \\
\hline Muscles + os de la cuisse (g) & $254^{\mathrm{b}}$ & $271^{\mathrm{a}}$ & $254^{\mathrm{b}}$ & $260^{\mathrm{b}}$ \\
\hline
\end{tabular}

a, b, c : effet significatif du croisement à $P<0,01$. 
Tableau 8. Héritabilité ( $\pm E T$ ) des caractères de qualité des magrets de canards mulards dans les populations de canes communes et de canards de Barbarie (Marie-Etancelin et al 2010).

\begin{tabular}{|l|c|c|}
\hline \multirow{2}{*}{} & \multicolumn{2}{|c|}{ Population } \\
\cline { 2 - 3 } & commune & Barbarie \\
\hline Muscle du magret & $0,32 \pm 0,04$ & $0,07 \pm 0,04$ \\
\hline Peau + gras du magret & $0,16 \pm 0,03$ & $0,10 \pm 0,04$ \\
\hline Lipides intramusculaires & $0,24 \pm 0,04$ & $0,11 \pm 0,04$ \\
\hline Exsudat sous vide & $0,02 \pm 0,02$ & $0,05 \pm 0,02$ \\
\hline Pertes à la cuisson & $0,03 \pm 0,02$ & $0,02 \pm 0,02$ \\
\hline pH 20 min post mortem & $0,04 \pm 0,03$ & $0,05 \pm 0,03$ \\
\hline pH ultime & $0,02 \pm 0,02$ & $0,02 \pm 0,02$ \\
\hline Force maximale de cisaillement & $0,06 \pm 0,03$ & $0,03 \pm 0,02$ \\
\hline Luminance (L*) & $0,12 \pm 0,04$ & $0,09 \pm 0,03$ \\
\hline Indice de rouge (a*) & $0,14 \pm 0,04$ & $0,14 \pm 0,04$ \\
\hline Indice de jaune (b*) & $0,13 \pm 0,04$ & $0,06 \pm 0,04$ \\
\hline
\end{tabular}

que celui du compartiment « peau + gras » (environ 0,15), la corrélation génétique entre les poids de ces deux compartiments étant faible (Poujardieu et al 1994b, Marie-Etancelin et al 2010). Le poids de la cuisse présente aussi une héritabilité intermédiaire, estimée à 0,33 chez le canard de Barbarie (Babilé 1989) et à 0,20 dans la voie maternelle du canard mulard (Marie-Etancelin et al 2010, tableau 8). Concernant la qualité physicochimique et technologique des magrets, seul le taux de lipides intramusculaires a une héritabilité supérieure à 0,20 . Hormis les trois paramètres de la couleur (héritabilités de l'ordre de 0,13 ), la plupart des autres caractères de qualité ont une très faible héritabilité (inférieure à 0,06 ). Néanmoins, les cinq QTL (zones des chromosomes expliquant une part importante de la variabilité génétique) les plus importants identifiés chez le canard mulard, concernent des caractères faiblement héritables, tels que les pertes à la cuisson, le $\mathrm{pH}$, la tendreté de la viande : ainsi, un QTL sur le chromosome 2 est associé aux pertes à la cuisson du tifs. Les principales différences entre les quatre types génétiques sont généralement dues aux effets additifs directs avec principalement des effets défavorables du canard de Barbarie sur la texture de la viande, qu'elle soit crue ou cuite.

A notre connaissance, aucun caractère de qualité de la viande n'est actuellement sélectionné chez le canard, hormis le poids du magret. Initialement sélectionné de façon indirecte via le poids du canard mulard à l'entrée en gavage (corrélation génétique de 0,78 avec le muscle du magret, Poujardieu et al 1994b), le poids du magret est à présent sélectionné directement dans la lignée commune à partir de mesures de son épaisseur chez le canard mulard (Marie-Etancelin et al 2008).

\section{Conclusion}

Les caractéristiques des carcasses et de la viande de palmipèdes gavés peuvent donc être très variables selon l'espèce (oie ou canard), le type génétique et le mode d'élevage et d'alimentation. Cette viande bénéficie d'une image positive dans l'esprit des consommateurs (Bouvarel et al 1998), en particulier pour ses qualités sensorielles liées à une teneur élevée en lipides. Le prix de la viande de canard est d'ailleurs proche de celui de la viande bovine à griller (Braine 2012). Afin que les consommateurs soient toujours satisfaits, la filière doit maîtriser les facteurs de variation de la qualité de la carcasse et de la viande. Jusqu'à présent, elle a toujours considéré la viande de palmipèdes gavés comme un sous-produit du foie gras, alors même que l'ensemble cuisses-pilons et magrets représente $50 \%$ de la valeur de la carcasse d'un canard mulard gavé à l'entrée en abattoir (Provialys, données non publiées). Si les facteurs zootechniques modifiant la quantité et la qualité des muscles, en particulier l'alimentation commencent à être bien connus, il reste des recherches à conduire sur les étapes de l'abattage et de la transformation, et probablement des innovations à réaliser sur les modes de présentation favorisant la praticité d'utilisation par les consommateurs et permettant à cette production de conserver un rythme de croissance positif. 


\section{Références}

AGPM, 2000. Elevage et gavage des canards mulards. Recueil technique. Editeur AGPM-TECHNIQUE, Paris, France, 24p.

Arroyo J., 2012. Influence de l'alimentation sur les performances des oies et la durabilité du système de production du foie gras : effets de la substitution du maïs par du sorgho et de la forme de présentation de l'aliment. Thèse de Doctorat, INP Toulouse, France, 329p.

Auvergne A., 1992. Facteurs de variation de la composition corporelle et tissulaire des canards avant et après gavage. Thèse de Doctorat d'Etat en Sciences, INP Toulouse, France, 252p.

Auvergne A., Dubois J.P., Verdier M., Babilé R., Sitko V., 1998. Conduite du gavage et caractéristiques technologiques et organoleptiques des produits d'oies grasses. $3^{\text {emes }}$ Journ. Rech. Palmipèdes à Foie Gras, Bordeaux, France, 115-118.

Auvergne A., Desforges M., Fernandez X., Gestes G., Babilé R., 2002. Croissance et performances de gavage de trois croisements de canards mulards. $5^{\text {emes }}$ Journ. Rech. Palmipèdes à Foie Gras, Pau, France, 46-49.

Babilé R., 1989. La production de foies gras de canards de Barbarie (Caïrina moschata) Aspects génétiques, nutritionnels et technologiques. Thèse de Doctorat, INP Toulouse, France, $231 \mathrm{p}$.

Babilé R., Auvergne A., Dubois J.P., Leprettre S., Manse H., 2000. Comparaison de différents systèmes de production de l'oie gavée : incidences sur la composition des muscles pectoraux et la qualité des magrets séchés. $4^{\text {emes }}$ Journ. Rech. Palmipèdes à Foie Gras, Arcachon, France, 151-154.

Baéza E., Rideau N., Chartrin P., Davail S., Hoo-Paris R., Mourot J., Guy G., Bernadet M.D., Juin H., Meteau K., Hermier D., 2005. Canards de Barbarie, Pékin et leurs hybrides : aptitude à l'engraissement. INRA Prod. Anim., 18, 131-141.

Baudonnet-Lenfant C., Auvergne A., Babilé R., 1991. Influence de la durée de jeûne avant l'abattage et du poids à la mise en gavage des canards de Barbarie sur la composition chimique hépatique. Ann. Zootech., 40, 161-170.

Bouvarel I., Castaing J., Zwick J.L., 1998. Le canard, une viande festive. Viandes et Produits Carnés, 19, 185-188.

Braine A., 2012. Le marché du canard à rôtir. Journée Nationale ITAVI des professionnels du canard à rôtir, 14/06/12, Angers, France, 13p.

Castaing J., Robin N., 1996. Influence de la céréale, maïs ou blé dans des aliments pour canards mulards. $2^{\text {èmes }}$ Journ. Rech. Palmipèdes à Foie Gras, Bordeaux, France, 57-60.

Castaing J., Robin N., 2000. Utilisation d'un maïs waxy pour le gavage à la pâtée. $4^{\text {èmes }}$ Journ. Rech. Palmipèdes à Foie Gras, Arcachon, France, 130-133.

Castaing J., Robin N., Peyhorgue A., Kesteloot R., Penven K., Bouvarel I., Latré L., 2000. Incidences des modalités d'élevage des canards mulards sur l'appréciation sensorielle des magrets. $4^{\text {èmes }}$ Journ. Rech. Palmipèdes à Foie Gras, Arcachon, France, 163-166.

Chapuis H., Larzul C., 2008. Comment estimer simultanément les paramètres génétiques des caractères de gavage dans les 2 lignées parentales du mulard en vue d'une sélection plus efficace $? 8^{\text {èmes }}$ Journ. Rech. Palmipèdes à Foie Gras, Arcachon, France, 29-32.

Chartrin P., Schiavone A., Bernadet M.D. Guy G., Mourot J., Duclos M.J., Baéza E., 2005. Effect of genotype and overfeeding on lipid deposition in myofibres and intramuscular adipocytes of breast and thigh muscles of ducks. Reprod. Nutr. Dev., 45, 87-99.

Chartrin P., Bernadet M.D., Guy G., Mourot J., Duclos M.J., Baéza E., 2006a. The effects of genotype and overfeeding on fat level and composition of adipose and muscle tissues in ducks. Anim. Res., 55, 231-244.

Chartrin P., Bernadet M.D., Guy G., Mourot J., Hocquette J.F., Rideau N., Duclos M.J., Baéza E., 2006b. Does overfeeding enhance genotype effects on energy metabolism and lipid deposition in breast muscle of ducks? Comp. Biochem. Physiol., Part A, 145, 413 418

Chartrin P., Meteau K., Juin H., Bernadet M.D., Guy G., Larzul C., Rémignon H. Mourot J., Duclos M.J., Baéza E., 2006c. Effects of intramuscular fat levels on sensory characteristics of duck breast meat. Poult. Sci., 85, 914-922.

Chartrin P., Bernadet M.D., Guy G., Mourot J., Hocquette J.F., Rideau N., Duclos M.J., Baéza E., 2007. Do age and feeding levels have comparable effects on fat deposition in breast muscle of mule ducks. Animal, 1, 113-123.

CIFOG, 2012. Rapport économique 2011. Assemblée Générale du 22/06/12, Saint-Palais, France, 75p.

Dubois J.P., Auvergne A., Babilé R., Verdier M., Lavigne F., 1993. L'alimentation des oies à gaver : optimisation de la conduite alimentaire et influence sur la croissance jusqu'à l'âge de 18 semaines (premiers résultats). $1^{\text {eres Journ }}$ Rech. Palmipèdes à Foie Gras, Bordeaux, France, 35-48.

Dubois J.P., Auvergne A., Babilé R., Verdier M., Leprettre S., Lavigne F., Vieillecroze D. 1996. Le point sur les facteurs d'amélioration de la production d'oies gavées. $2^{\text {èmes }}$ Journ. Rech. Palmipèdes à Foie Gras, Bordeaux, France, 89-92.

Dubois J.P., Lavigne F., Leprettre S., Auvergne A., Babilé R., 2000. Incidence du taux d'incorporation de grains dans le mélange de gavage sur les performances de l'oie gavée. $4^{\text {èmes }}$ Journ. Rech. Palmipèdes à Foie Gras, Arcachon, France, 113-116.

Ducamp C., Guy G., Skiba F., Coudure R., 2008. Comparaison de deux modes de conservation du maïs pour le gavage de canards mulards mâles à la pâtée : maïs sec/maïs humide inerté. $8^{\text {èmes }}$ Journ. Rech. Palmipèdes à Foie Gras, Arcachon, France, 185-188.

Fernandez X., Lahirigoyen E., Auvergne A., Molette C., Bouillier-Oudot M., 2010. The effects of stunning methods on product qualities in force fed ducks and geese. 1. Carcass downgrading and meat quality. Animal, 4 128-138.

Fernandez X., Bouillier-Oudot M., Molette C., Bernadet M.D., Manse H., 2011. Duration of transport and holding in lairage at constant postprandial delay to slaughter - Effects on fatty liver and breast muscle quality in mule ducks. Poult. Sci., 90, 2360-2369.
Gabarrou J.F., Babilé R., Auvergne A., Manse H., Bouillier-Oudot M., Everlet P. Chambert S., 2000. Modification du profil d'acides gras des tissus du canard mulard par incorporation d'huiles lors du gavage au grain.

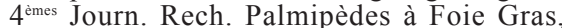
Arcachon, France, 155-158.

Guy G., Rousselot-Pailley D., Gourichon D., 1995. Comparaison des performances de l'oie, du canard mulard et du canard de Barbarie soumis au gavage. Ann. Zootech., 44, 297-305.

Guy G., Hermier D., Davail S., Bely M., André J.M., Hoo-Paris R., 1999. Meat production and force-feeding ability of different types of ducks. In: $1^{\text {st }}$ World Waterfowl Conf., 1-4/12/99, Taichung, Taiwan, 462-468.

Guy G., Doussan I., Gouraud P., 2000. Forme de présentation et valorisation du maïs chez le canard mulard gavé. $4^{\text {èmes }}$ Journ. Rech. Palmipèdes à Foie Gras, Arcachon, France, 159-162.

Kileh-Wais M., Elsen J.M., Vignal A., Feves K., Vignoles F., Fernandez X., Manse H., Davail S., André J.M., Bastianelli D., Bonnal L., Filangi O., Baéza E., Guémené D., Genet C., Bernadet M.D., Dubos F., Marie-Etancelin C., 2013. Detection of QTL controlling metabolism, meat quality, and liver quality traits of the overfed interspecific hybrid mule duck. J. Anim. Sci., 91, 588-604.

Larzul C., Rouvier R., Rousselot-Pailley D. Guy G., 2000. Estimation of genetic parameters for growth, carcass and overfeeding traits in a white geese strain. Genet. Sel. Evol., 32, 415 427.

Larzul C., Imbert B., Bernadet M.D., Guy G., Rémignon H., 2006. Meat quality in an intergeneric factorial crossbreeding between Muscovy (Cairina moschata) and Pekin (Anas platyrhyncos) ducks. Anim. Res., 55, 1-11.

Leprettre S., Dubois J.P., Auvergne A., Babilé R., Lavigne F., Sitko V., 1998. Elevage des oies : influence de la concentration énergétique de l'aliment en démarrage sur les performances de croissance, de découpe et de gavage. $3^{\text {emes }}$ Journ. Rech. Palmipèdes à Foie Gras, Bordeaux, France, 83-86.

Leprettre S., Dubois J.P., Lavigne F., Babilé R., 2000a. Densité en élevage et performances de croissance chez l'oie destinée au gavage. $4^{\text {èmes }}$ Journ. Rech. Palmipèdes à Foie Gras, Arcachon, France, 109-112.

Leprettre S., Auvergne A., Dubois J.P., Babilé R., Lavigne F., 2000b. Systèmes d'élevage des oies et performances de croissance. $4^{\text {èmes }}$ Journ. Rech. Palmipèdes à Foie Gras, Arcachon, France, 105-108.

Litt J., 2010a. Le réseau ferme de références palmipèdes à foie gras : résultats 2008 des producteurs de l'échantillon constant en filière longue. $10^{\text {èmes }}$ Journ. Rech. Palmipèdes à Foie Gras, Bordeaux, France, 231-235

Litt J., 2010b. Comparaison de différents modes de logements en gavage : influence sur le produit, le bien-être animal et les conditions de travail. $10^{\text {èmes }}$ Journ. Rech. Palmipèdes à Foie Gras, Bordeaux, France, 203-206.

Marie-Etancelin C., Chapuis H., Brun J.M. Larzul C., Richard M.M., Rouvier R., 2008. Genetics and selection of mule ducks in France : A review. World Poult. Sci. J., 64, 187 207. 
Marie-Etancelin C., Basso B., Davail S., Gontier K., Fernandez X., Vitezica Z.G., Bastianelli D., Baéza E., Bernadet M.D., Guy G., Brun J.M., Legarra A., 2010. Genetic parameters of product quality and hepatic metabolism in fattened mule ducks. J. Anim. Sci., 89, 669-679.

Peillod C., Coudure R., Skiba F., Laborde M., 2010. Gavage au maïs humide conservé par inertage : détermination des effets de la durée de stockage du maïs en silo souple sur les performances zootechniques des canards mulards mâles. $10^{\text {èmes }}$ Journ. Rech. Palmipèdes à Foie Gras, Bordeaux, France, 61-64.

Peyhorgue A., Robin N., Castaing J., Bouvarel I., Latré L., 2002a. Qualité des magrets de canards mulards en relation avec la durée de gavage. $5^{\text {emes }}$ Journ. Rech. Palmipèdes à Foie Gras, Pau, France, 144-147.

Peyhorgue A., Robin N., Castaing J., Bouvarel I., 2002b. Caractéristiques des magrets de canards mulards en relation avec un apport d'huile végétale en gavage. $5^{\text {emes }}$ Journ. Rech. Palmipèdes à Foie Gras, Pau, France, 156-159.

Poujardieu B., Rouvier R., Rousselot-Pailley D., Guy G., Rosinski A., Wezyk S., 1994a. Croissance et aptitude au gavage d'oies de 3 génotypes. Ann. Zootech., 43, 197-211.

Poujardieu B., Guichard F., Laventure P., 1994b. Paramètres génétiques de croissance et de gavage de la cane commune. Genet. Sel. Evol., 26, 468-72.

Richard M.M., Marie-Etancelin C., Dubos F., Bernadet M.D., Guy G., Brun J.M., 2006. Genetic parameters of reproduction and force- feeding traits in geese under artificial lighting conditions. $8^{\text {th }}$ World Cong. Appl. Livest. Prod. Instituto Prociencias Minas Gerais, Minas Gerais, Belo Horizonte, Brésil, 407-425.

Robin N., Castaing J., 1993. Influence des apports azotés en élevage sur les performances des canards mulards. $1^{\text {ires }}$ Journ. Rech. Palmipèdes à Foie Gras, Bordeaux, France, 51-66.

Robin N., Castaing J., 1998a. Effets d'une restriction alimentaire en élevage sur les performances des canards mulards. $3^{\text {emes }}$ Journ. Rech. Palmipèdes à Foie Gras, Bordeaux, France, 91-94.

Robin N., Castaing J., 1998b. Itinéraires techniques et identification des produits du gavage de canards mulards. $3^{\text {imes }}$ Journ. Rech. Palmipèdes à Foie Gras, Bordeaux, France, 123-126.

Robin N. Castaing J, 1998c. Incidence d'une supplémentation énergétique et protéique du maïs broyé sur la production de foie gras de canards mulards en gavage. $3^{\text {èmes }}$ Journ. Rech. Palmipèdes à Foie Gras, Bordeaux, France, 95-98.

Robin N., Castaing J., 2000. Incidence du type de grain de maïs pour le gavage du canard mulard. $4^{\text {emes }}$ Journ. Rech. Palmipèdes à Foie Gras, Arcachon, France, 126-129.

Robin N., Sazy E., Castaing J., 2000. Modes de logement du canard mulard en gavage observations de gavage et performances zootechniques. $4^{\text {èmes }}$ Journ. Rech. Palmipèdes à Foie Gras, Arcachon, France, 134-137.

Robin N., Larroudé P., Peyhorgue A., Castaing J., 2002. Etude comparative de quatre types génétiques pour une production de canards mulards lourds. $5^{\text {emes }}$ Journ. Rech. Palmipèdes à Foie Gras, Pau, France, 41-45.

Sazy E., Héraut F., Robin N., 1993. Evolution de la composition corporelle des canards mulards pendant le gavage. $1^{\text {eres }}$ Journ. Rech. Palmipèdes à Foie Gras, Bordeaux, France, 47-61.

Sazy E. Héraut F., 1996a. Résultats comparatifs en élevage et gavage de mulards selon le type de père. $2^{\text {èmes }}$ Journ. Rech. Palmipèdes à Foie Gras, Bordeaux, France, 37-40.

Sazy E., Héraut F., 1996b. Evolution du poids vif du canard mulard mâle au cours du jeûne. $2^{\text {emes }}$ Journ. Rech. Palmipèdes à Foie Gras, Bordeaux, France, 53-56.

Setiawan I., 1994. Etude comparative de différents types génétiques de canards mulards : croissance, composition corporelle et tissulaire, et qualité des produits obtenus avant et après gavage. Thèse de Doctorat, INP Toulouse, $270 \mathrm{p}$.

Tai C., Rouvier R., 1998. Crossbreeding effect on sexual dimorphism of body weight in intergeneric hybrids obtained between Muscovy and Pekin duck. Genet. Sel. Evol., $30,163-170$

Zanusso J., Rémignon H., Guy G., Manse H., Babilé R., 2002. Effets du gavage sur les caractéristiques des fibres musculaires et sur le métabolisme du muscle pectoral (Pectoralis major) chez le canard de Barbarie. $5^{\text {emes }}$ Journ. Rech. Palmipèdes à Foie Gras, Pau, France, 160-163.

\section{Résumé}

La viande de palmipèdes gavés se distingue de la viande de palmipèdes non gavés surtout par son enrichissement en lipides (teneur multipliée par deux) qui modifie la qualité nutritionnelle et sensorielle des produits et leur aptitude à la conservation. Toutefois, ces caractéristiques peuvent fortement varier selon l'itinéraire technique mis en ouvre pendant les phases d'élevage et de gavage et en particulier en fonction de la composition et des modalités de distribution de l'aliment, de l'âge à la mise en gavage et de sa durée. Les étapes de pré-abattage et la technologie d'abattage et de transformation sont également importantes à considérer. Les paramètres génétiques de la qualité de la carcasse et de la viande ont surtout été étudiés chez le canard. Ces critères de qualité sont dans l'ensemble faiblement héritables. Récemment, plusieurs QTL ont été mis en évidence en particulier pour les pertes à la cuisson, le pH et la texture de la viande. La connaissance des gènes impliqués dans le contrôle de ces paramètres devrait permettre à plus ou moins long terme de les inclure dans les schémas de sélection.

\section{Abstract}

\section{Carcass and meat quality of overfed waterfowl}

The meat of overfed ducks and geese is quite different from that of waterfowl fed ad libitum, particularly because of its higher lipid content (2-fold more) affecting the nutritional and sensorial quality of products and their storage ability. However, these characteristics can be quite variable according to the technical conditions used during rearing and overfeeding periods such as the composition and distribution modalities of diets, age at the beginning of the overfeeding period and duration of overfeeding. The pre-slaughter conditions and the slaughtering and processing technologies are also important. The genetic parameters of carcass and meat quality traits have been mainly evaluated for ducks. Meat quality traits are weakly heritable. Recently, few QTL have been highlighted particularly for cooking loss, $\mathrm{pH}$ and meat texture. The knowledge of the genes involved in the control of these parameters should in the short or long term allow including them in selection programmes.

BAÉZA E., FERNANDEZ X., MARIE-ETANCELIN C., 2013. Qualité des carcasses et de la viande des palmipèdes gavés. In : Palmipèdes à foie gras. Fortun-Lamothe L. (Ed). Dossier, INRA Prod. Anim., 26, 425-434. 